\title{
Non-Akhiezer-Polovin Model on Plasma Electrostatic Wave and Electron Beam
}

\author{
H. Lin $^{*}$
}

State Key Laboratory of High Field Laser Physics, Shanghai Institute of Optics and Fine Mechanics, P. O. Box 800-211, Shanghai 201800, China

\begin{abstract}
We study, beyond the well-known Akhiezer-Polovin model, plasma electrostatic wave and unmagnetized electron beam.Our investigation is based on a stricter theory in which a long-lasting misconception about zerotemperature fluid motion equation is removed. Our theory explains some authors' puzzle about why a narrowly focused (charged particles) beam is preserved in the presence of strong space charge forces. The interaction of such a unmagnetized charged particles beam with a plasma electrostatic wave is studied in details and some universal results are revealed. These exact information are crucial to accurate estimation of the quality of a plasma wakefield and hence its performance in acceleration.
\end{abstract}

Keywords: PACS: 41.75.-i, 29.27.Bd, 29.27.Fh, 52.27.Jt, 52.35.-g, 52.35.Fp, 52.35.Mw, 52.35.Sb, 52.65.-y.

\section{INTRODUCTION}

Among numerous theoretical investigations on plasmabased particle acceleration [1-16], Akhiezer-Polovin (A-P) model is a popular description on zero-temperature plasma electrostatic (ES) wave $[3-5,9,15]$. According to this model, there is a convective term appearing in the fluid motion equation in the zero-temperature limit.

$\partial_{t} p(u)+u \cdot \nabla_{r} p(u)=-[E+u \times B]$

where $u$ is fluid velocity, $p(u)=\frac{u}{\sqrt{1-u^{2}}}$ and $u \cdot \nabla_{r} p(u)$ is the so-called convective term. Because the fluid motion equation in the zero-temperature limit and 4 Maxwell equations (Meqs) form a basis for studying macroscopic property of charged particles, it is very crucial to ensure these equations being correct or strictly derived from microscopic Vlasov-Maxwell (V-M) theory, which is also the theoretical basis of beam physics [17-27].

The derivation of the A-P model is completely a standard textbook procedure. By deriving a finite-temperature fluid motion equation [28] from Vlasov equation (VE) and then putting this equation in the zero-temperature limit, we can discard the thermal-pressure term and finally obtain Eq. (1). This procedure seems to be perfect. Eq. (1) can also be derived from other way [see later]. Agreement between different derivation methods further confirms Eq. (1) to be strictly correct.

However, we cannot ignore a deep inconsistency behind the above-described standard procedure of deriving the

*Address correspondence to this author at the State Key Laboratory of High Field Laser Physics, Shanghai Institute of Optics and Fine Mechanics, P. O. Box 800-211, Shanghai 201800, China; E-mail: linhai@siom.ac.cn
Eq. (1). That is, when putting the finite-temperature fluid motion equation in the zero-temperature limit, we actually have subconsciously believed that zero-temperature type distribution is a strict solution of the VE. This belief might be the fundamental reason for a long-lasting misconception popular in plasma and beam physics. We have pointed out this inconsistency elsewhere [31]. For convenience of readers, we also introduce them in details in this work.

This forces us, beyond the A-P model, to re-consider ES wave, electron beam and various aperiodic ES structures in charged particles. (here, wave is a periodic "structure". Because studied physical quantities are not bound to be periodic, we introduce a more general term "structure"). This will be done in Sec.II. The interaction between periodic structure and aperiodic one is the content of the third subsection of Sec.II. The 4-th subsection is for phase space coherent structure. Section. III is a brief summary.

\section{THEORY}

\subsection{Starting Model Equations}

We start from well-known V-M equation set [28, 29]

$\left[\partial_{t}+v \cdot \nabla-[E+v \times B] \cdot \partial_{p(v)}\right] f=0$,

$\partial_{t} E=n u+\nabla \times B$

$\nabla \cdot E=-n+Z N_{i}$

$\nabla \times E=-\partial_{t} B$

$\nabla \cdot B=0$.

where $p(v)=m_{e} \frac{v}{\sqrt{1-v \cdot v}}, n=\int f d^{3} v, u=\frac{\int v f d^{3} v}{\int f d^{3} v}$ and $m_{e}$ is the static mass of an electron. Moreover, electron charge $e$ 
has been absorbed into $E$ and $B$. Moreover, all equations are expressed in term of Euler variables $(r, t)$, and $v$ is independent of $r$ and $t$.

A strict analysis have revealed that if $f(r, v, t)$ is a strict solution of the VE, $f_{0}=f\left(r, \frac{\int v f d^{3} v}{\int f d^{3} v}, t\right) * \delta\left(v-\frac{\int v f d^{3} v}{\int f d^{3} v}\right)$ is not $[31,32]$. This result can be found from more general Klimontovich-Dupree (K-D) theory [28]. According to the K-D theory, a particle system can be described by a function $N(r, v, t)=\sum_{i} \delta\left(r_{t}(t)-r\right) \delta\left(d_{t} r_{i}(t)-v\right)$ which meets the VE. Obviously, a function with more constraint $N_{0}(r, v, t)=$ $\sum_{i} \delta\left(r_{t}(t)-r\right) \delta\left(d_{t} r_{i}(t)-v\right) \delta(v-u(r, t))$ will correspond to a zero-temperature type distribution function. Even though $N(r, v, t)=\sum_{i} \delta\left(r_{t}(t)-r\right) \delta\left(d_{t} r_{i}(t)-v\right)$ always meets the VE, $\quad N_{0}(r, v, t)=\sum_{i} \delta\left(r_{t}(t)-r\right) \delta\left(d_{t} r_{i}(t)-v\right) \delta(v-u(r, t))$ does not. This can be directly verified by substituting $N_{0}$ back into the VE.

For any particle system described by a microscopic distribution function $f$, we can always view it as the summation of two subsystems of a same fluid velocity $u$, one consists of all particles whose velocities are equal to $u=\frac{\int v f d^{3} v}{\int f d^{3} v}$ and the other is described by a "hollow" distribution $f_{h o}$ which meets $f_{h o}(r, v=u, t)=0$ and $u=\frac{\int v f_{h o} d^{3} v}{\int f_{h o} d^{3} v}$. Above results reveal that each subsystem does not have a conserved total particle number and hence exchange particles with the other. This might be the fundamental reason for why the zero-temperature type subsystem, which corresponds to $f_{0}=f-f_{h o}$, does not meet the VE.

The microscopic dynamics equation of $f_{0}=n_{0}(r, t) \delta(v-u(r, t))$, where $n_{0}=\int f_{0} d^{3} v$, can be derived straightforward [31]. According to strict theory [31, $32]$, the continuity equation associated with $n_{0}$ becomes

$\partial_{t} n_{0}+u \cdot \nabla_{r} n_{0}=0$,

rather than our familiar $\partial_{t} n_{0}+u \cdot \nabla_{r} n_{0}=-n_{0} \nabla_{r} \cdot u$ (i.e. $\left.\partial_{t} n_{0}+\nabla_{r} \cdot\left(n_{0} u\right)=0\right)$. This reflects the subsystem described by $f_{0}$ having particle exchange with other. Namely, because $E$ is space-time dependent, a charged particle system cannot be at zero-temperature state in which at any space position, all particles have a same velocity. Space-inhomogeneous $E$ will lead to, in some space positions, the temperature differing from 0 and hence thermal spread in particles' velocities appearing (which means some particles being out of the kernel group described by $f_{0}$ and into the hollow group described by $f-f_{0}$ ).

Likewise, a macroscopic fluid motion equation can be derived [31, 32]

$\partial_{t} \frac{u}{\sqrt{1-u^{2}}}+E+u \times B=0$.

In contrast, we can, from the VE, derive our familiar fluid motion equation (for arbitrary temperature) $\partial_{t} u+\frac{\int(v-u) \nabla_{r}[v f] d^{3} v}{n}+\frac{\int[E(r, t)+v \times B(r, t)] \cdot\left[\sqrt{1-v^{2}}\right]^{3} * f d^{3} v}{n}=0$.

Obviously, two strict equations of $u$ suggest a balance relation

$\frac{\int(v-u) \nabla_{r}[v f] d^{3} v}{n}=$

$\left[-\frac{\int[E(r, t)+v \times B(r, t)] \cdot\left[\sqrt{1-v^{2}}\right]^{3} * f d^{3} v}{n}+[E+u \times B]\left(\sqrt{1-u^{2}}\right)^{3}\right]$,

which is an implicit equation of the temperature or the thermal spread.

Above discussions have displayed in details, from microscopic theory, why Eq. (1) is inaccurate. On the other hand, Eq. (1) can also be derived completely via macroscopic fluid theory $[20,21]$. This forces us to analyze what is misunderstood when deriving Eq. (1) from fluid theory.

Because in standard fluid theory, if a physical field is expressed by Lagrangian variable $Q(\tilde{r}(t), t)$, its total differential with respect to $t$ will be strictly $d_{t} Q(\tilde{r}(t), t)=$ $\partial_{t} Q(\tilde{r}(t), t)+d_{t} \tilde{r} \cdot \nabla_{\tilde{r}} Q(\tilde{r}(t), t)$. Thus, if $Q$ stands for momentum $p$ there will be $\partial_{t} Q(\tilde{r}(t), t)+d_{t} \tilde{r} \cdot \nabla_{\tilde{r}} Q(\tilde{r}(t), t)=d_{t} Q(\tilde{r}(t), t)=$

$F(\tilde{r}(t), t)$ (where $F(\tilde{r}(t), t)$ stands for the field of force). For this equation, people often, by the relation $d_{t} \tilde{r} \equiv$ $u(\tilde{r}(t), t)$ which defines the trajectory of a fluid element, rewrite it as a more familiar form $F(\tilde{r}(t), t)=\partial_{t} p(\tilde{r}(t), t)+$ $u(\tilde{r}(t), t) \cdot \nabla_{\tilde{r}} p(\tilde{r}(t), t)=\partial_{t} p(u(\tilde{r}(t), t))+u(\tilde{r}(t), t) \cdot$

$\nabla_{\tilde{r}} p(u(\tilde{r}(t), t))$, where $p(u)=\frac{u}{\sqrt{1-u \cdot u}}$. Then, after transforming this familiar form from an expression in term of Lagrangian variables $(\tilde{r}(t), t)$ to that in term of Euler variable $(r, t)$, we will obtain Eq. (1).

However, if noting that the relation $d_{t} \tilde{r} \equiv u(\tilde{r}(t), t)$ can lead to 3 equivalent expressions of $d_{t} \tilde{r} \cdot \nabla_{\tilde{r}} p(u(\tilde{r}(t), t)): 1$. $u(\tilde{r}(t), t) \cdot \nabla_{\tilde{r}} p(u(\tilde{r}(t), t))$ (merely replacing $d_{t} \tilde{r}$ left to $\cdot$ operator with $u(\tilde{r}(t), t)$ ); 2. $d_{t} \tilde{r} \cdot \nabla_{\tilde{r}} p\left(d_{t} \tilde{r}\right)$ (merely replacing $u(\tilde{r}(t), t)$ with $\left.d_{t} \tilde{r}\right) ; 3 . u(\tilde{r}(t), t) \cdot \nabla_{\tilde{r}} p\left(d_{t} \tilde{r}\right)$. Namely, the relation $d_{t} \tilde{r} \equiv u(\tilde{r}(t), t)$ will lead to $d_{t} \tilde{r} \cdot \nabla_{\tilde{r}} p(u(\tilde{r}(t), t)) \equiv u(\tilde{r}(t), t) \cdot \nabla_{\tilde{r}} p(u(\tilde{r}(t), t)) \equiv d_{t} \tilde{r} \cdot$ $\nabla_{\tilde{r}} p\left(d_{t} \tilde{r}\right) \equiv u(\tilde{r}(t), t) \cdot \nabla_{\tilde{r}} p\left(d_{t} \tilde{r}\right)$. Most essentially, the property $\quad d_{t} \tilde{r} \cdot \nabla_{\tilde{r}} p\left(d_{t} \tilde{r}\right)=\left.d_{t} \tilde{r} \cdot \frac{d p(\lambda)}{d \lambda}\right|_{\lambda=d_{t} \tilde{r}} \nabla_{\tilde{r}} d_{t} \tilde{r} \quad \equiv 0$ (because of $\nabla_{\tilde{r}} d_{t} \tilde{r} \equiv 0$ ) determines all these 4 expressions to be $\equiv 0$. In other words, the so-called convective term $u(\tilde{r}(t), t) \cdot \nabla_{\tilde{r}} p(u(\tilde{r}(t), t))$ is indeed 0 . More detailed demonstration are presented in an appendix.

Clearly, if merely using the relation $d_{t} \tilde{r} \equiv u(\tilde{r}(t), t)$ but on purpose ignoring the fact that $u(\tilde{r}(t), t) \cdot \nabla_{\tilde{r}} p(u(\tilde{r}(t), t))$ is indeed $\equiv 0$, we can find that $d_{t} p(u(\tilde{r}(t), t))=F(\tilde{r}(t), t)$ will agree with $\partial_{t} p(u(r, t))+u(r, t) \cdot \nabla_{\tilde{r}} p(u(r, t))=$ $F(r, t)$. Incomplete/partial utilization of the relation $d_{t} \tilde{r} \equiv u(\tilde{r}(t), t)$ can yield a different agreement. 
In mathematical language, incomplete/partial utilization will lead to extraneous root of the equation set

$F(\tilde{r}(t), t)=d_{t} p\left(d_{t} \tilde{r}\right)$;

$d_{t} \tilde{r} \equiv u(\tilde{r}(t), t)$.

i.e. the solution of $\partial_{t} p(u(r, t))+u(r, t) \cdot \nabla_{\tilde{r}} p(u(r, t))=$ $F(r, t)$ cannot meet Eqs. $(11,12)$ but the solution of Eqs. (11, 12) can meet $\partial_{t} p(u(r, t))+u(r, t) \cdot \nabla_{\tilde{r}} p(u(r, t))=F(r, t)$.

In short, by analyzing on what condition Eqs. $(11,12)$ have a common solution of $\tilde{r}(t)$, we can find this demanding a relation between two functionals $F(r, t)=\partial_{t} p(u(r, t))$,i.e. Eq. (8).

\subsection{Macroscopic ES Structures in Neutral and Non- Neutral Plasmas}

For different ES structures, we wish to find related solutions which are static in a moving frame of a constant velocity $\frac{1}{\eta} c$. Therefore, we introduce following definitions

$\xi=\eta z / c-t ; p=\frac{u}{\sqrt{1-[u / c]^{2}}} ; u(z, t, r, \theta)=u(\xi, r, \theta)$,

where $r, \theta$ and $z$ are coordinates in the cylindric frame.

\subsubsection{ES Wave}

Detailed analysis reveals that the ES wave in 3-D case corresponds to an equation (where $\beta=\frac{p_{r}}{p_{z}}$ and $\lambda=\frac{p_{\theta}}{p_{z}}, p_{z}$ is dimensionless momentum) [30]

$\left[1-\frac{\eta\left(1+\beta^{2}+\lambda^{2}\right) p_{z}}{\sqrt{1+\left(1+\beta^{2}+\lambda^{2}\right) p_{z}^{2}}}\right] \partial_{\xi \xi} p_{z}=-Z N_{i} \frac{p_{z}}{\sqrt{1+\left(1+\beta^{2}+\lambda^{2}\right) p_{z}^{2}}}$,

which corresponds to a first integral of general form

$\left(\partial_{\xi} p_{z}\right)^{2}+f_{0}\left(r, \theta, p_{z}\right)=G(r, \theta)$,

where $G(r, \theta)$ is a binary function of $r$ and $\theta$, and $f_{0}$ stands for well-known Sagdeev potential.

The relation between $p_{z}$ and density profile $n$ reads [30]

$n=\frac{\sqrt{1+\left(1+\beta^{2}+\lambda^{2}\right) p_{Z}^{2}}}{\sqrt{1+\left(1+\beta^{2}+\lambda^{2}\right) p_{z}^{2}}-\eta\left(1+\beta^{2}+\lambda^{2}\right) p_{z}} Z N_{i}$.

Note that the condition $n t \geq 0$ will lead to a constraint on $p_{z}$

$\sqrt{1+\left(1+\beta^{2}+\lambda^{2}\right) p_{z}^{2}}-\eta\left(1+\beta^{2}+\lambda^{2}\right) p_{z}>0$,

or $p_{z}<\frac{1}{\sqrt{\eta^{2}\left(1+\beta^{2}+\lambda^{2}\right)^{2}-\left(1+\beta^{2}+\lambda^{2}\right)}}<\frac{1}{\sqrt{\eta^{2}-1}}$ if $\eta>1$.

$f_{0}\left(r, \theta, p_{z}\right)$ and $G(r, \theta)$ in Eq. (15) read [30]

$f_{0}\left(r, \theta, p_{z}\right)=$

$\frac{2}{c}\left\{\begin{array}{l}-\sqrt{1+\left(1+\beta^{2}+\lambda^{2}\right) p_{z}^{2}}-\frac{1}{2} \frac{\eta\left(1+\beta^{2}+\lambda^{2}\right)}{\sqrt{c}} \ln \frac{\sqrt{1+\left(1+\beta^{2}+\lambda^{2}\right) p_{z}^{2}}-\frac{\eta\left(1+\beta^{2}+\lambda^{2}\right)}{\sqrt{c}}}{\sqrt{1+\left(1+\beta^{2}+\lambda^{2}\right) p_{z}^{2}}+\frac{\eta\left(1+\beta^{2}+\lambda^{2}\right)}{\sqrt{c}}} \\ -\eta\left(1+\beta^{2}+\lambda^{2}\right) p_{z}+\frac{1}{2} \frac{\eta\left(1+\beta^{2}+\lambda^{2}\right)}{\sqrt{c}} \ln \frac{\sqrt{c} p_{z}-1}{\sqrt{c} p_{z}+1}\end{array}\right\} Z N_{i}$

(19)
$G(r, \theta)=f_{0}\left(r, \theta, p_{z}=0\right)+\left.\left(\partial_{\xi} p_{z}\right)^{2}\right|_{p_{z}=0}$,

where

$c=\left[\eta^{2}\left(1+\beta^{2}+\lambda^{2}\right)^{2}-\left(1+\beta^{2}+\lambda^{2}\right)\right]>0$ if $\eta>1$.

In the A-P model [5], $\partial_{\xi}[\eta p-\Gamma]=E$ is used and $u$ is a function of $\eta p-\Gamma$ (where $\Gamma=\sqrt{1+p^{2}}, \xi=z-\frac{1}{\eta} c t$ and $\eta$ is a constant). A first integral of $\eta p-\Gamma$ (which is denoted by $W$ ) is thus derived (where $Z$ is ionic charge and $N_{i}$ is ionic density).

$\left(\partial_{\xi} W\right)^{2}+\frac{Z N_{i}}{\left(\eta^{2}-1\right)}\left[\eta \sqrt{\left(W^{2}+\eta^{2}\right)-1} \pm W\right]=$ const

Following similar procedure, we use $\partial_{\xi} \eta p=E$ and derive a first integral of $p$.

$\left(\partial_{\xi} p\right)^{2}+\frac{Z N_{i}}{3 \eta}\left[\left(\sqrt{p^{2}+1}\right)^{3}+p^{3}\right]=$ const

As shown in Fig. (1), under same value of $\left[\left.\partial_{\xi} p\right|_{\xi=0},\left.p\right|_{\xi=0}\right]$, the behaviors of $p$ and $E$ from different models are somewhat different. Luckily, there is no marked difference between the magnitudes of two shapes. Also, two shapes are almost of a same wavelength. Actually, even though some authors have studied 1-D ES wave beyond the A-P model [6], they seem to be not aware that their treatment is indeed rigorous rather than an approximation, and hence underestimate the importance of their works.

\subsubsection{Unmagnetized Charged Particles Beam}

Same procedure can yield similar formulas about the unmagnetized electron beam

$\left[1-\frac{\eta\left(1+\beta^{2}+\lambda^{2}\right) p_{z}}{\sqrt{1+\left(1+\beta^{2}+\lambda^{2}\right) p_{z}^{2}}}\right] \partial_{\xi \xi} p_{z}=$
$\left\{\left[\left(\partial_{r} \beta+\frac{\beta}{r}+\frac{1}{r} \partial_{\theta} \lambda\right) \partial_{\xi} p_{z}\right]\right\} \frac{p_{z}}{\sqrt{1+\left(1+\beta^{2}+\lambda^{2}\right) p_{z}^{2}}}$.

which implies

$\partial_{\xi} p_{z}-\left(\partial_{r} \beta+\frac{\beta}{r}+\frac{1}{r} \partial_{\theta} \lambda\right) f_{0}\left(r, \theta, p_{z}\right)=G(r, \theta)$.

Likewise, $n$ reads

$$
\begin{aligned}
& n=-\left(\partial_{r} \beta+\frac{\beta}{r}+\frac{1}{r} \partial_{\theta} \lambda\right) \partial_{\xi} p_{z}-\eta\left(1+\beta^{2}+\lambda^{2}\right) \partial_{\xi \xi} p_{z} \\
& =-\frac{\sqrt{1+\left(1+\beta^{2}+\lambda^{2}\right) p_{z}^{2}}}{\sqrt{1+\left(1+\beta^{2}+\lambda^{2}\right) p_{z}^{2}}-\eta\left(1+\beta^{2}+\lambda^{2}\right) p_{z}}\left(\partial_{r} \beta+\frac{\beta}{r}\right. \\
& \left.+\frac{1}{r} \partial_{\theta} \lambda\right) \partial_{\xi} p_{z} \\
& =-\frac{\sqrt{1+\left(1+\beta^{2}+\lambda^{2}\right) p_{z}^{2}}}{\sqrt{1+\left(1+\beta^{2}+\lambda^{2}\right) p_{z}^{2}}-\eta\left(1+\beta^{2}+\lambda^{2}\right) p_{z}} \\
& {\left[\left(\partial_{r} \beta+\frac{\beta}{r}+\frac{1}{r} \partial_{\theta} \lambda\right)^{2} f_{0}\left(r, \theta, p_{z}\right)+\left(\partial_{r} \beta+\frac{\beta}{r}+\frac{1}{r} \partial_{\theta} \lambda\right) G(r, \theta)\right] .}
\end{aligned}
$$

It is well-known that such a general form

$f_{2}(y) y^{\prime \prime}+f_{1}(y) * y^{\prime}+f_{0}(y)=0$

which contains a linear term of $y^{\prime}$, cannot correspond to a first integral unless $f_{1}(y)=0$. According to Eq. (24) and 


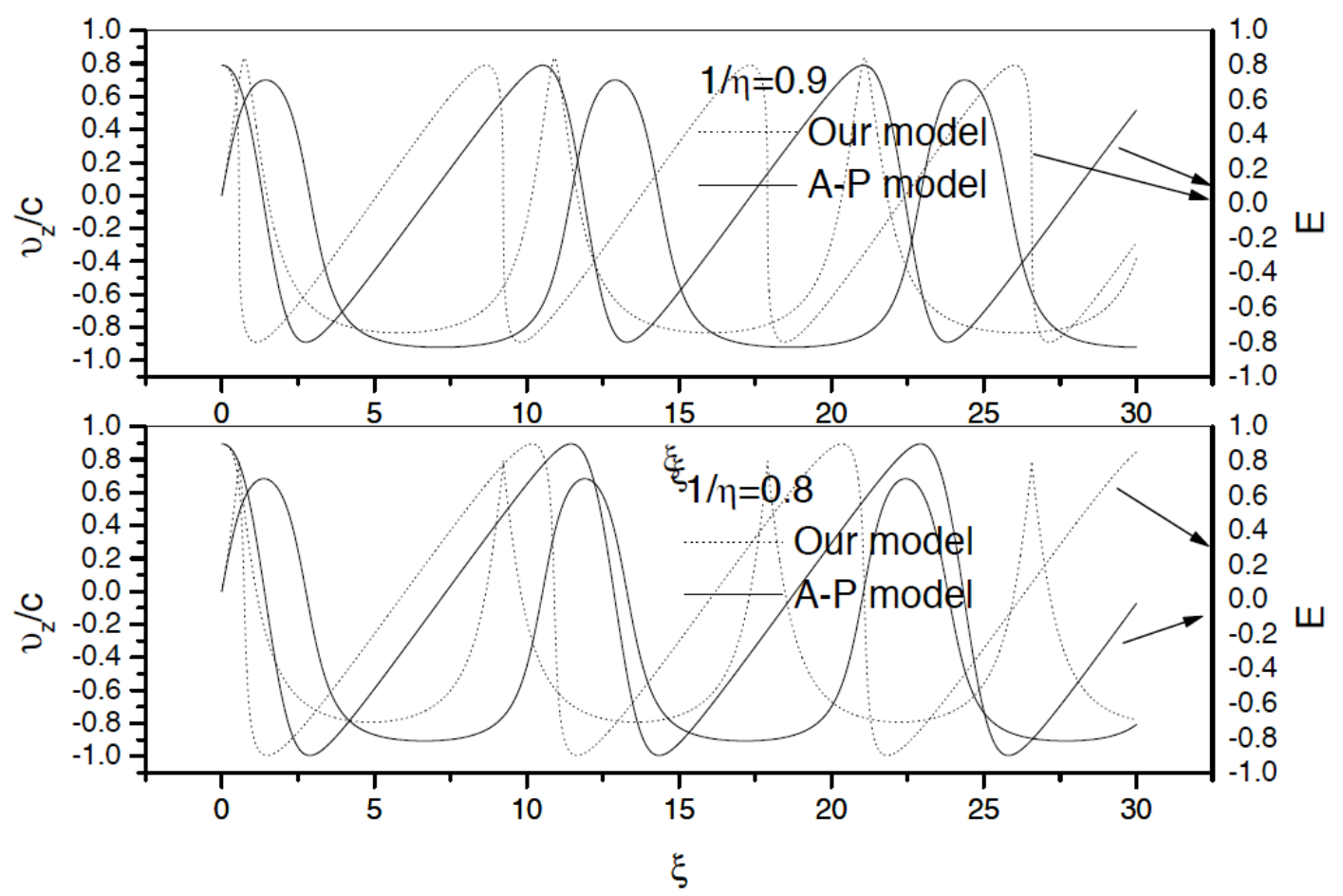

Fig. (1). The behaviors of $p$ and $E \operatorname{VS} \xi=z-\frac{c}{\eta} t$, where $\frac{c}{\eta}$ is the phase velocity of the ES wave and $Z N_{i}=1$ is choosen.

Eq. (26), an unmagnetized electron beam is impossible to be described by a periodic solution because this will imply $\partial_{r} \beta+\frac{\beta}{r}+\frac{1}{r} \partial_{\theta} \lambda=0$ and $n=0$ anywhere. Thus, an unmagnetized electron beam must have

$\partial_{r} \beta+\frac{\beta}{r}+\frac{1}{r} \partial_{\theta} \lambda \neq 0$

Note that there should be $n \geq 0$ anywhere. This will lead to a constraint on $p_{z}$

$\frac{\left[\left(\partial_{r} \beta+\frac{\beta}{r}+\frac{1}{r} \partial_{\theta} \lambda\right)^{2} f_{0}\left(r, \theta, p_{Z}\right)+\left(\partial_{r} \beta+\frac{\beta}{r}+\frac{1}{r} \partial_{\theta} \lambda\right) G(r, \theta)\right]}{\sqrt{1+\left(1+\beta^{2}+\lambda^{2}\right) p_{Z}^{2}}-\eta\left(1+\beta^{2}+\lambda^{2}\right) p_{Z}} \notin \leq 0$

$f_{0}\left(r, \theta, p_{z}\right)$ and $G(r, \theta)$ in Eq. (25) read

$f_{0}\left(r, \theta, p_{z}\right)=$
$\frac{1}{c}\left\{\begin{array}{l}-\sqrt{1+\left(1+\beta^{2}+\lambda^{2}\right) p_{z}^{2}}-\frac{1}{2} \frac{\eta\left(1+\beta^{2}+\lambda^{2}\right)}{\sqrt{c}} \ln \frac{\sqrt{1+\left(1+\beta^{2}+\lambda^{2}\right) p_{z}^{2}}-\frac{\eta\left(1+\beta^{2}+\lambda^{2}\right)}{\sqrt{c}}}{\sqrt{1+\left(1+\beta^{2}+\lambda^{2}\right) p_{z}^{2}}+\frac{\eta\left(1+\beta^{2}+\lambda^{2}\right)}{\sqrt{c}}} \\ -\eta\left(1+\beta^{2}+\lambda^{2}\right) p_{z}+\frac{1}{2} \frac{\eta\left(1+\beta^{2}+\lambda^{2}\right)}{\sqrt{c}} \ln \frac{\sqrt{c} p_{z}-1}{\sqrt{c} p_{z}+1}\end{array}\right\}$

(30)

$G(r, \theta)=-\left(\partial_{r} \beta+\frac{\beta}{r}+\frac{1}{r} \partial_{\theta} \lambda\right) f_{0}\left(r, \theta, p_{z}=\frac{1 / \eta}{\sqrt{1-(1 / \eta)^{2}}}\right)$,

where $c=\left[\eta^{2}\left(1+\beta^{2}+\lambda^{2}\right)^{2}-\left(1+\beta^{2}+\lambda^{2}\right)\right]>0$ if $\eta>1$.

Here, the reason for why $G(r, \theta)$ has above expression is for following consideration: the $p_{z}$-value at the position meeting $\partial_{t} p_{z}=E=0$ is equal to $\frac{1 / \eta}{\sqrt{1-(1 / \eta)^{2}}}$ and thus $1 / \eta$ is a characteristic constant velocity of such an ES structure. Eq. (30) indicates that for $p_{z}>0, f_{0}\left(r, \theta, p_{z}\right)$ decreases with $p_{z}$ increasing. Correspondingly, the constraint Eq. (29) will become

$$
\begin{aligned}
& \sqrt{1+\left(1+\beta^{2}+\lambda^{2}\right) p_{z}^{2}}-\eta\left(1+\beta^{2}+\lambda^{2}\right) p_{z} \oplus \leq 0 \\
& \operatorname{or} p_{z}+\frac{1}{\sqrt{\eta^{2}\left(1+\beta^{2}+\lambda^{2}\right)^{2}-\left(1+\beta^{2}+\lambda^{2}\right)}} \text { if } \eta>1 .
\end{aligned}
$$

According to Eqs. $(26,29,30,31), p_{z}>\frac{1}{\sqrt{\eta^{2}-1}}$ will correspond to $n<0$. Thus, for a unmagnetized beam, its $p_{z}$ is confined within a regime

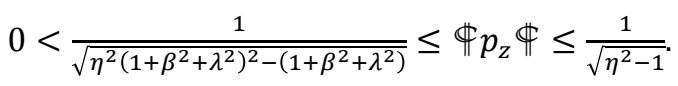

Some examples of $p_{z}$-profile and corresponding $n$-profile are presented in Fig. (2). Transverse boundary conditions are dependent on longitudinal one through $\beta$ and $\lambda$. Here, we plot these curves in similar shapes. For example, $p_{z}$ rises monotonically to $\frac{1}{\sqrt{\eta^{2}-1}}$ and $n$ drops monotonically to 0 . This same trend just reflects these curves are from a common equation with different parameter values. The effect of related parameter values are exhibited by different ranges of coordinate regime. Note that at $r=0.01$ (see Fig. (2b)), $p_{z}$ rises from 0.5773334 to 0.5773350 over a regime $0<\xi<0.025$. In contrast, at $r=10, p_{z}$ rises from 0.025 to 0.35 over a regime $0<\xi<0.030$. Such a difference due to different $r$-values could also be found from $n$-curves. At larger $r$ regime, $n$ drops relatively gently from a value, which is far below that at smaller $r$ regime, to 0 . From those $n$-curves at different $r$-values, one could find that for a given $\xi, n$ is inverse proportional to $r$. 

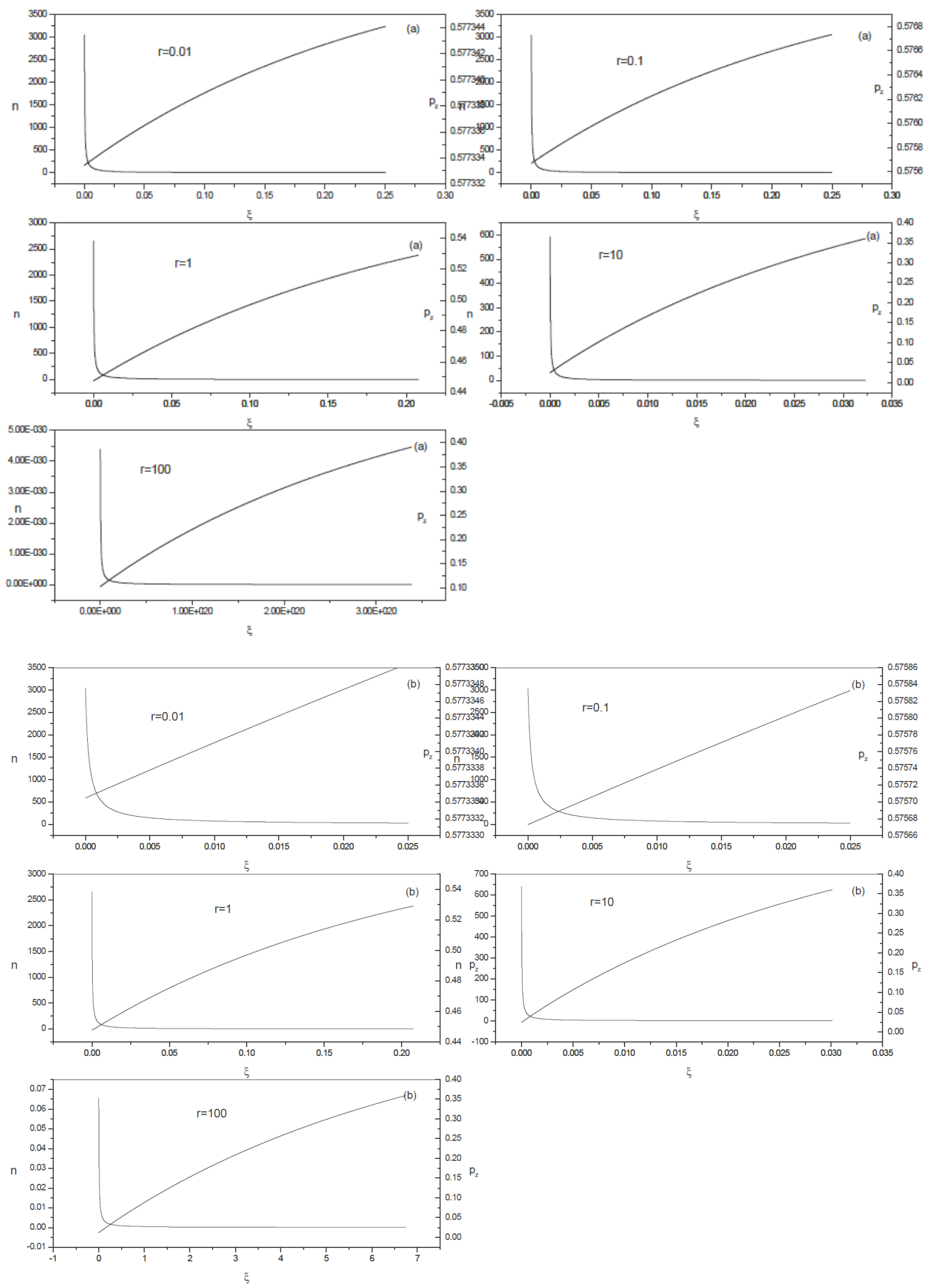

Fig. (2). Examples of density profile ( $n$-profile) of a unmagnetized electron beam and corresponding $p_{z}$-profile, where $\eta=1 / 2, \partial_{r} \beta+\frac{\beta}{r}=$ $\exp \left(-r^{2} / R_{\perp}^{2}\right)$ with $R_{\perp}=20$ in (a) and $\partial_{r} \beta+\frac{\beta}{r}=\frac{R_{\perp}^{2}}{R_{\perp}^{2}+r^{2}}$ with $R_{\perp}=20$ in (b). Moreover, corresponding bondary conditions for Eq. (23) are $\left.p_{z}\right|_{\xi=0}=0$ and $\left.\partial_{z} p_{z}\right|_{\xi=0}=-0.01$. Here, $p_{z}$ is in unit of $m_{e} c, n$ is in unit of $\frac{8.85 * 0.511}{1.6} * 10^{7}[\mu m]^{-3}, E$ is in unit of $0.511 * 10^{6}[\mathrm{Volt} / \mu \mathrm{m}]$, and $R$ is in unit of $\mu \mathrm{m}$. 
Above theoretical and numerical results indicate that even though all particles in the beam have a same charge, their collective self-consistent fields could confine themselves to form a globally-moving, small-volume and hard-core charged "cloud". Here, the word "hard-core" refers to that the density at the inside region of the cloud is far larger than that at the outside region.. A correct knowledge on a charged particles beam is very valuable to correctly understand its interaction with matter. As pointed out by Chao et al. [26], it is quite counterintuitively to understand that such a narrowly focused (charged particles) beam is preserved in the presence of strong space charge forces. Our above theory could answer this doubt to some extent.

\subsection{Beam-wave Interaction}

When a unmagnetized electron beam is injected into a pre-existing plasma ES wave, their respective self-electric fields will strongly perturb each other. As shown previously (for example, Eq. (24)), every ES structure is usually described by its phase velocity $\frac{1}{7} c$ and shape (which is determined by the boundary condition). Before making detailed study, we first discuss the phase velocity.

\subsubsection{Phase Velocity}

From Eq. (8) at $B \equiv 0$ case, we know that $u$-value at the zero point of a moving $E$ just corresponds to a constant characteristic velocity. Considering that the phase velocity $\frac{1}{\eta} c$ is also a a constant characteristic velocity, for convenience, we choose two constant characteristic velocities being equal and hence $\frac{1}{\eta} c$ is defined as the fluid velocity at the zero point of the moving $(E, B)$ fields.

For every ES structure, its $P(u)$-profile and $E$-profile are power series of $z-\int_{0}^{t} S\left(t^{\prime}\right) d t^{\prime}$

$E=\sum_{i \dagger 0} c E_{i}\left(z-\int_{0}^{t} S\left(t^{\prime}\right) d t^{\prime}\right)^{i} ; P(u)=\sum_{i \dagger 0} c P u_{i}(z-$

$\left.\int_{0}^{t} S\left(t^{\prime}\right) d t^{\prime}\right)^{i}$.

where $S=\frac{1}{\eta}$ and $c P u_{0}=\frac{S}{\sqrt{1-S^{2}}}$. Note that because $\partial_{t} P(u)=-E$, there is $c E_{0}=0$ if $d_{t} S=0$. Obviously, for an ES structure free from perturbation, there are $d_{t} S=0$ and $c E_{0}=0$. Namely, along a trajectory defined by $P(z, t)=$ $\frac{S}{\sqrt{1-S^{2}}}$, there is always $E(z, t)=0$. Thus, $[P(z, t), E(z, t)]=$ $\left[\frac{S}{\sqrt{1-S^{2}}}, 0\right]$ defines a phase-velocity section of an ES structure, or $z=\int_{0}^{t} S\left(t^{\prime}\right) d t^{\prime}$ section in which $E\left(\int_{0}^{t} S\left(t^{\prime}\right) d t^{\prime}, t\right)=0$ exists.

\subsubsection{Momentum Exchange}

When two structures $A$ and $B$ encounter, their phasevelocity sections are not always at a same position. Namely, at the phase-velocity section of $A$ structure, there might be $E_{B} \neq 0$ and vice versa. Thus, a momentum exchange will occur and can be described by

$$
\begin{aligned}
& -d_{t} \frac{S_{A}}{\sqrt{1-S_{A}^{2}}}=-d_{t} p_{A}\left(\int_{0}^{t} S_{A}\left(t^{\prime}\right) d t^{\prime}, t\right) \\
& \quad=E_{A}\left(\int_{0}^{t} S_{A}\left(t^{\prime}\right) d t^{\prime}, t\right)+E_{B}\left(\int_{0}^{t} S_{A}\left(t^{\prime}\right) d t^{\prime}, t\right) \\
& =E_{B}\left(\int_{0}^{t} S_{A}\left(t^{\prime}\right) d t^{\prime}, t\right)=\sum_{i \dagger \geq 0} c E B_{i} *\left(\int_{0}^{t} S_{A}\left(t^{\prime}\right) d t^{\prime}-\right. \\
& \left.\int_{0}^{t} S_{B}\left(t^{\prime}\right) d t^{\prime}\right)^{i} ; \\
& -d_{t} \frac{S_{B}}{\sqrt{1-S_{B}^{2}}}=-d_{t} p_{B}\left(\int_{0}^{t} S_{B}\left(t^{\prime}\right) d t^{\prime}, t\right) \\
& =E_{A}\left(\int_{0}^{t} S_{B}\left(t^{\prime}\right) d t^{\prime}, t\right) \\
& +E_{B}\left(\int_{0}^{t} S_{B}\left(t^{\prime}\right) d t^{\prime}, t\right) \\
& =E_{A}\left(\int_{0}^{t} S_{B}\left(t^{\prime}\right) d t^{\prime}, t\right)=\sum_{i \dagger \geq 0} c E A_{i} *\left(\int_{0}^{t} S_{B}\left(t^{\prime}\right) d t^{\prime}-\right. \\
& \left.\int_{0}^{t} S_{A}\left(t^{\prime}\right) d t^{\prime}\right){ }^{i},
\end{aligned}
$$

which could be written as

$$
\begin{aligned}
& d_{t t} \frac{S_{A}}{\sqrt{1-S_{A}^{2}}}=-\left[S_{A}-S_{B}\right] * \sum_{i \uparrow \geq 1} c E B_{i} *\left(\int_{0}^{t} S_{A}\left(t^{\prime}\right) d t^{\prime}-\right. \\
& \left.\int_{0}^{t} S_{B}\left(t^{\prime}\right) d t^{\prime}\right)^{i-1} ; \\
& d_{t t} \frac{S_{B}}{\sqrt{1-S_{B}^{2}}}=-\left[S_{B}-S_{A}\right] * \sum_{i \uparrow \geq 1} c E A_{i} *\left(\int_{0}^{t} S_{B}\left(t^{\prime}\right) d t^{\prime}-\right. \\
& \left.\int_{0}^{t} S_{A}\left(t^{\prime}\right) d t^{\prime}\right)^{i-1} .
\end{aligned}
$$

Some examples of the solutions of Eqs. $(39,40)$ are presented in Fig. (3), which indicates $\max \left(S_{A}, S_{B}\right)$ decreasing and $\min \left(S_{A}, S_{B}\right)$ increasing and hence a momentum exchange between two structures. Such an exchange will continue until $S_{A}-S_{B}$ becoming 0 and imply two structures being merged into a new structure. Note that the relative ratio between $c E A_{i}$ and $c E B_{i}$ affects the saturated value of $S$. Moreover, how soon to arrive at this saturated value is determined by the values of $c E A_{i}$ and $c E B_{i}$.

\subsubsection{Shape Distortion}

A periodic structure can be described by a first integral of $p_{z}$ and its $\beta$-profile is restricted to meet $\partial_{r} \beta+\frac{\beta}{r}+\frac{1}{r} \partial_{\theta} \lambda=0$, while an aperiodic one cannot and its $\beta$-profile is restricted to meet $\partial_{r} \beta+\frac{\beta}{r}+\frac{1}{r} \partial_{\theta} \lambda \neq 0$. If $A$ is aperiodic and $B$ is periodic, their different detailed features in shapes determine that even in the merged new structure $A+B$, two subsystems still affect each other. Noting that the fluid velocity of this $A+B$ structure is 


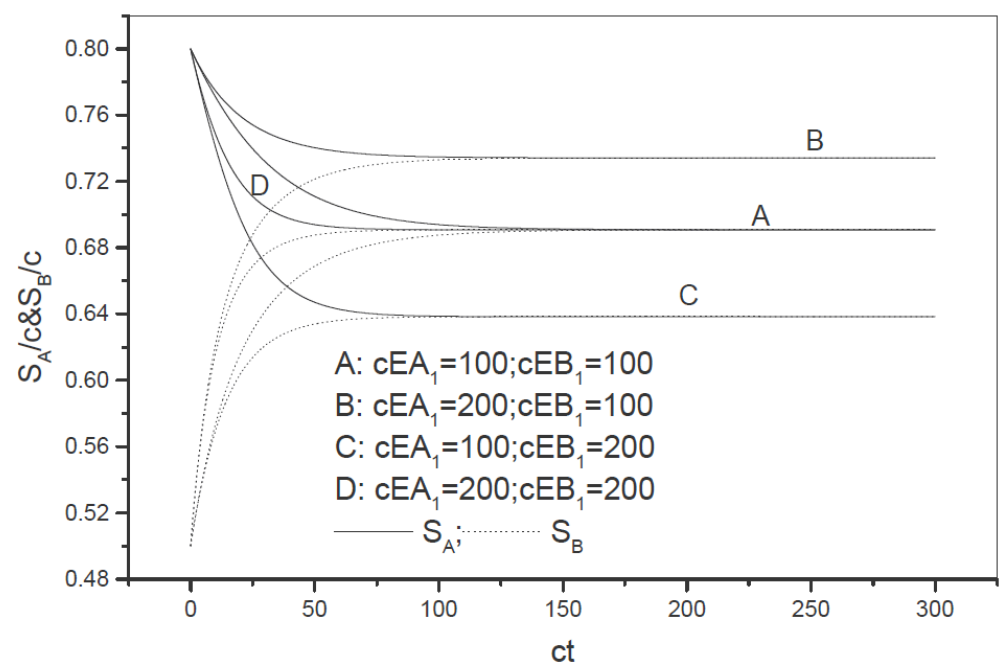

Fig. (3). Examples of the histories of $S_{A}$ and $S_{B}$, where $c E A_{1}$, as well as $c E B_{1}$, is in unit of $0.511 * 10^{6}[\mathrm{Volt} / \mu \mathrm{m}] / \mu \mathrm{m}$.

$u_{A+B}=\frac{n_{A} u_{A}+n_{B} u_{B}}{n_{A}+n_{B}}$.

Moreover, from

$\partial_{t} p\left(u_{B}\right)=\partial_{t} p\left(u_{A}\right)=\partial_{t} p\left(u_{A+B}\right)=-E$,

we have

$p\left(u_{A}\right)-p\left(u_{A+B}\right)=C_{A}(r, \theta, z)$;

$p\left(u_{B}\right)-p\left(u_{A+B}\right)=C_{B}(r, \theta, z)$,

where $C_{A}(r, \theta, z)$ and $C_{B}(r, \theta, z)$ are both $t$-independent.

$>$ From initial condition, we can find that $\beta_{A+B}=$ $\frac{\left[n_{A} u_{A}+n_{B} u_{B}\right]_{r}}{\left[n_{A} u_{A}+n_{B} u_{B}\right]_{z}}$, as well as $\lambda_{A+B}=\frac{\left[n_{A} u_{A}+n_{B} u_{B}\right]_{\theta}}{\left[n_{A} u_{A}+n_{B} u_{B}\right]_{z}}$, usually does not meet $\partial_{r} \beta+\frac{\beta}{r}+\frac{1}{r} \partial_{\theta} \lambda=0$. Therefore, $p_{z}\left(u_{A+B}\right)$ will be aperiodic and cannot correspond to a first integral. Following the same procedure of deriving Eq. (14) and (24), we obtain a similar equation for $p_{Z}\left(u_{A+B}\right)$

$\left[1-\frac{\eta\left(1+\beta_{A+B}^{2}+\lambda_{A+B}^{2}\right) p_{z}}{\sqrt{1+\left(1+\beta_{A+B}^{2}+\lambda_{A+B}^{2}\right) p_{Z}^{2}}}\right] \partial_{\xi \xi} p_{Z}$

$=\left\{\left[\left(\partial_{r} \beta_{A+B}+\frac{\beta_{A+B}}{r}+\frac{1}{r} \partial_{\theta} \lambda_{A+B}\right) \partial_{\xi} p_{z}\right]-Z N_{i}\right\}$

$\frac{p_{Z}}{\sqrt{1+\left(1+\beta_{A+B}^{2}+\lambda_{A+B}^{2}\right) p_{Z}^{2}}}$

After solving $p_{Z}\left(u_{A+B}\right)$ from this equation, we could know $p\left(u_{A}\right)$ and $p\left(u_{B}\right)$ according to Eqs. $(43,44)$. Once the histories of $p\left(u_{A}\right)$ and $p\left(u_{B}\right)$ are known, the evolutions of $n_{A}$ and $n_{B}$ can be strictly calculated from respective continuity equations.

Therefore, when two ES structures encounter, their interaction are displayed by two aspects. As shown in Eqs.(39, 40), their phase velocities are approaching to a common value. On the other hand, their respective shapes are also distorted. For instance, Eq. (45) indicates that the shape of a periodic structure is distorted because of another aperiodic structure.

\subsection{Phase Space Structure}

First, we consider $B \neq 0$ case. The VE can be written as

$0=\left[\partial_{t}+v \cdot \nabla-[E+v \times B] \cdot \partial_{p(v)}\right] f$

$=\left[\partial_{t}-E \cdot \partial_{p(v)}\right] f+v \cdot\left[\nabla-B \times \partial_{p(v)}\right] f$.

Moreover, if $f$ is a strict solution of the VE, any monovariable function of $f$, or $g(f)$, is also a strict solution.

For the case in which $E$ and $B$ are running waves of a phase velocity $\frac{1}{\eta} c$, i.e. $E=E\left(r-\frac{1}{\eta} c t\right)$ and $B=B(r-$ $\frac{1}{\eta} c t$ ), we should note a relation between $E$ and $B: E=$ $-\frac{1}{\eta} c \times B+\nabla \Phi\left(r-\frac{1}{\eta} c t\right)$, which arises from $\nabla \times E=-\partial_{t} B$. Here, $\Phi\left(r-\frac{1}{\eta} c t\right)$ is a scalar function but cannot be simply taken as ES potential (because $-\frac{1}{\eta} c \times B$ also has divergence or $\left.\nabla \cdot\left(-\frac{1}{\eta} c \times B\right)=\frac{1}{\eta} c \cdot \nabla \times B \neq 0\right)$. In this case, the VE can be further written as

$$
\begin{aligned}
& 0=\left[\partial_{t}-E \cdot \partial_{p(v)}\right] f+v \cdot\left[\nabla-B \times \partial_{p(v)}\right] f \\
& =\left[\partial_{t}+\frac{1}{\eta} c \times B \cdot \partial_{p(v)}\right] f+v \cdot\left[\nabla-B \times \partial_{p(v)}\right] f-\nabla \Phi \cdot \partial_{p(v)} f \\
& =\left[\partial_{t}+\frac{1}{\eta} c \cdot B \times \partial_{p(v)}\right] f+v \cdot\left[\nabla-B \times \partial_{p(v)}\right] f-\nabla \Phi \cdot \partial_{p(v)} f \\
& =\left(v-\frac{1}{\eta} c\right) \cdot\left[\nabla-B \times \partial_{p(v)}\right] f-\nabla \Phi \cdot \partial_{p(v)} f .
\end{aligned}
$$

It is easy to verify that any function of $p+\int E(r-$ $\left.\frac{1}{\eta} c t\right) d t$ will meet

$0=\left[\partial_{t}-E \cdot \partial_{p(v)}\right] g\left(p+\int E\left(r-\frac{1}{\eta} c t\right) d t\right)$ 


$$
\begin{aligned}
& =\left[-\frac{1}{\eta} c \cdot \nabla+\frac{1}{\eta} c \cdot B \times \partial_{p(v)}\right] g \\
& =-\frac{1}{\eta} c \cdot\left[\nabla-B \times \partial_{p(v)}\right] g
\end{aligned}
$$

where we have used the property $\partial_{t} \int E\left(r-\frac{1}{\eta} c t\right) d t=$ $-\frac{1}{\eta} c \cdot \nabla \int E\left(r-\frac{1}{\eta} c t\right) d t$. Thus, if $\nabla \Phi \equiv 0$, any monovariable function of $p+\int E\left(r-\frac{1}{\eta} c t\right) d t$, or $g\left(p+\int E(r-\right.$ $\left.\frac{1}{\eta} c t\right) d t$ ), will be a strict solution of the VE.

On the other hand, for more general $\nabla \Phi$, we can find that any mono-variable function of $\sqrt{1+\frac{p^{2}}{c^{2}}}-\frac{1}{\eta} c \cdot p+\Phi$, or $g\left(\sqrt{1+\frac{p^{2}}{c^{2}}}-\frac{1}{\eta} c \cdot p+\Phi\right)$, is a strict solution of the VE. According to Eq.(47), $\partial_{p}\left[\sqrt{1+\frac{p^{2}}{c^{2}}}-\frac{1}{\eta} c \cdot p\right]$ will contribute a vector parallel to $\left(v-\frac{1}{\eta} c\right)$ and hence make the operator $\left(v-\frac{1}{\eta} c\right) \cdot B \times \partial_{p(v)}$ has zero contribution.

Therefore, for coherent self-consistent fields $E=E(r-$ $\left.\frac{1}{\eta} c t\right)$ and $B=B\left(r-\frac{1}{\eta} c t\right)$, the phase space distribution, if $\nabla \Phi \equiv 0$, can be described by a positive-valued function of $p+\int E\left(r-\frac{1}{\eta} c t\right) d t$, for example exp $\left[-\left(p+\int E(r-\right.\right.$ $\left.\left.\left.\frac{1}{\eta} c t\right) d t\right)^{2}\right], \sin ^{2}\left(\exp \left[-\left(p+\int E\left(r-\frac{1}{\eta} c t\right) d t\right)^{2}\right]\right)$, etc. We can further pick out reasonable solutions according to the definition of $u$

$u=\frac{\int \frac{p}{\sqrt{1+p^{2}}} g\left(p+\int E\left(r-\frac{1}{\eta} c t\right) d t\right) d^{3} p}{\int g\left(p+\int E\left(r-\frac{1}{\eta} c t\right) d t\right) d^{3} p}$.

Likewise, same procedure exists for more general $\nabla \Phi$ and $g\left(\sqrt{1+\frac{p^{2}}{c^{2}}}-\frac{1}{\eta} c \cdot p+\Phi\right)$.

Actually, a set of macroscopic functions $(E, B, u)$ can have multiple microscopic solutions of corresponding VE. Therefore, usually we know the phase space distribution from the initial condition of the microscopic distribution $f(r, p, t=0)$. From the function dependence of $f(r, p, t=$ 0 ) on $p$, we can obtain the function dependence of $g$ on $\sqrt{1+\frac{p^{2}}{c^{2}}}-\frac{1}{\eta} c \cdot p$ and hence determine detailed function form of $g$.

Detailed procedure of determining function form of $g$ is described as follows [32]: We can seek for special space position $R$ in which $E(R, 0)=-\frac{1}{\eta} c \times B(R, 0)$, or $\left.\nabla \Phi(r, 0)\right|_{r=R}=0$, exists. The initial distribution at $R$, i.e., $f(R, p, t=0)$, is thus a mono-variable function $p$. At the same time, two expressions are equivalent and hence there is
$f(R, p, t=0)=g(K+\Phi(R, 0))=g(K)$. where $K=\sqrt{1+\frac{p^{2}}{c^{2}}}-$ $\frac{1}{\eta} c \cdot p$ and $\Phi(R, 0)=0$ (if $\left.\nabla \Phi(r, 0)\right|_{r=R}=0$ ). Because of certain relation between $p$ and $K$, once the expansion coefficients $c_{i}$ in $f(R, p, t=0)=\sum_{i} c_{i} p^{i}$ is known, the expansion coefficients $d_{i}$ in $g(K)=\sum_{i} d_{i} K^{i}$ is also easy to be calculated.

Then, we consider $B \equiv 0$ case. Clearly, BGK modes [7, 19] are analytic strict solutions of the VE in $B \equiv 0$ case

$0=\left[\partial_{t}-E\left(r-\frac{1}{\eta} c t\right) \cdot \partial_{p(v)}\right] f+v \cdot \nabla f$,

whose solutions are mono-variable functions of $\phi(r-$ $\left.\frac{1}{\eta} c t\right)+\sqrt{1+p^{2}}-\frac{1}{\eta} c \cdot p$, where $\phi\left(r-\frac{1}{\eta} c t\right)$ is scalar potential and $E=-\nabla \phi$. Moreover, there is a similar procedure of determining function form of $g$.

We should note that $K$ is a nonlinear function of $v$ and the maximum value of $K$, or $K_{\max }$ is reached at $v=\frac{1}{\eta} c$. Thus, even $g$ is a Dirac function of $K+\Phi, g$ cannot be a Dirac function of $v$, i.e. $g \sim \delta(v-u(r, t))$. The nonlinear function relation between $K$ and $v$ determines that $g$ is at least a summation of two Dirac components: $g=f_{1}(r, t) \delta\left(v-u_{1}(r, t)\right)+f_{2}(r, t) \delta\left(v-u_{2}(r, t)\right)+\ldots$ This agree with previous conclusion that functions of a general form $f_{1}(r, t) \delta\left(v-u_{1}(r, t)\right)$ cannot meet VE.

We should also note that, because of nonlinear function relation between $g$ and $K+\Phi$, the maximum of $g$, or $g_{\text {max }}$, is usually reached at $K+\Phi \neq K_{\max }+\Phi$. Namely, if $g_{\max }$ is reached at $K=K_{g \max }$, this $K_{g \max }$ usually corresponds to two values of $v$. In contrast, $K_{\max }$ merely corresponds to a value of $v$. Thus, the contour plot of $g$ in the phase space will take on complicated structures, such as hole, island etc. Some examples of such a complicated phase space structure is shown in Fig. (4).

\section{SUMMARY}

By a universal equation set of $(E, B, u)$, we study the 3-D nonlinear plasma ES wave beyond the A-P model. The first integral of the nonlinear wave are presented. Also, we explain why an unmagnetized charged particles beam must have aperiodic density profile. The interaction between a periodic ES structure and an aperiodic one are studied by this universal equation set. Moreover, we also present a standard procedure for constructing strict solution of VE. China.

This work is supported by National Science Fund of

\section{APPENDIX}

The fluid velocity is usually defined as

$u\left(r_{i}(t), t\right) \equiv \frac{\sum_{m} \delta\left(r_{i}(t)-r_{m}(t)\right) d_{t} r_{m}(t)}{\sum_{m} \delta\left(r_{i}(t)-r_{m}(t)\right)}$. 


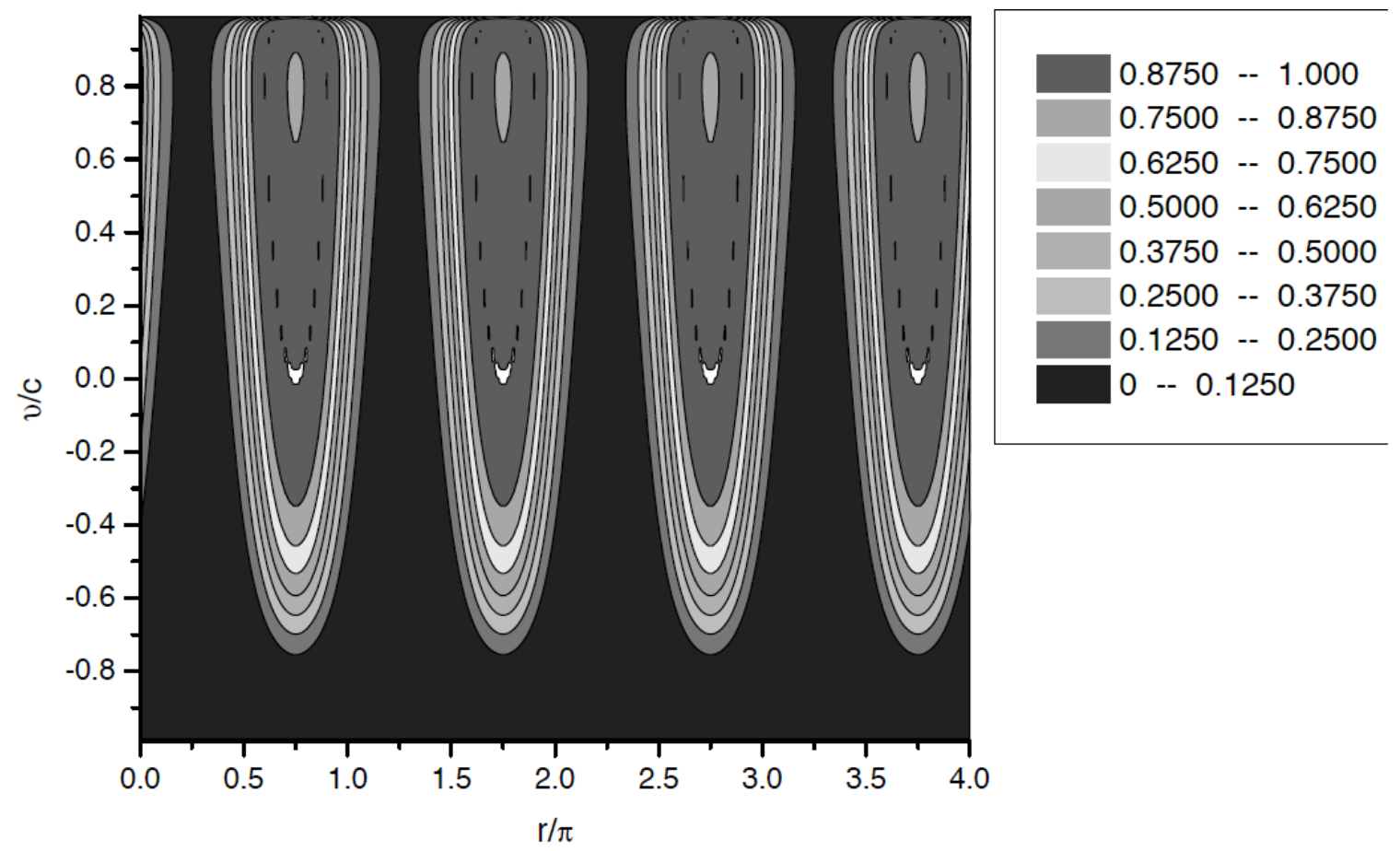

Fig. (4). Example of hole structure and island structure in the phase space contour plot of normalized microscopic distribution, where the self-consistent fields are periodic and have a phase velocity $0.8 c$.

We can also use a definition $R V$

$R V\left(r_{i}(t), t\right) \equiv \sum_{m} \delta\left(r_{i}(t)-r_{m}(t)\right) *\left[d_{t} r_{m}(t)-d_{t} r_{i}(t)\right]$

and re-write $u\left(r_{i}(t), t\right)$ as

$$
\begin{aligned}
& u\left(r_{i}(t), t\right) \equiv \frac{\sum_{m} \delta\left(r_{i}(t)-r_{m}(t)\right) *\left[d_{t} r_{m}(t)-d_{t} r_{i}(t)\right]}{\sum_{m} \delta\left(r_{i}(t)-r_{m}(t)\right)}+d_{t} r_{i}(t) \\
&= \frac{R\left(r_{i}(t), t\right)}{\sum_{m} \delta\left(r_{i}(t)-r_{m}(t)\right)}+d_{t} r_{i}(t) . \\
& \text { Clearly, there is } \\
& \nabla_{r_{i}} u\left(r_{i}(t), t\right) \\
&= \frac{\sum_{m} \sum_{n}\left[\nabla_{r_{i}} \delta\left(r_{i}(t)-r_{m}(t)\right)\right] * \delta\left(r_{i}(t)-r_{n}(t)\right) *\left[d_{t} r_{m}(t)-d_{t} r_{n}(t)\right]}{\left[\sum_{m} \delta\left(r_{i}(t)-r_{m}(t)\right)\right]^{2}} \\
&= \frac{-\sum_{m} \sum_{n}\left[\nabla_{r_{m}} \delta\left(r_{i}(t)-r_{m}(t)\right)\right] * \delta\left(r_{i}(t)-r_{n}(t)\right) *\left[d_{t} r_{m}(t)-d_{t} r_{n}(t)\right]}{\left[\sum_{m} \delta\left(r_{i}(t)-r_{m}(t)\right)\right]^{2}} \\
&= \frac{-\sum_{m} \sum_{n} \nabla_{r_{m}}\left\{\delta\left(r_{i}(t)-r_{m}(t)\right) * \delta\left(r_{i}(t)-r_{n}(t)\right) *\left[d_{t} r_{m}(t)-d_{t} r_{n}(t)\right]\right\}}{\left[\sum_{m} \delta\left(r_{i}(t)-r_{m}(t)\right)\right]^{2}} \\
&= \frac{-\sum_{m} \nabla_{r_{m}}\left\{\delta ( r _ { i } ( t ) - r _ { m } ( t ) ) * \sum _ { n } \left\{\delta\left(r_{i}(t)-r_{n}(t)\right) *\right.\right.}{\left.\left.\left[d_{t} r_{m}(t)-d_{t} r_{n}(t)\right]\right\}\right\}} \\
&= \frac{-\sum_{m} \nabla_{r_{m}}\left\{\delta\left(r_{i}(t)-r_{m}(t)\right) *\left[d_{t} r_{m}(t)-d_{t} r_{i}(t)\right] * \sum_{n} \delta\left(r_{i}(t)-r_{n}(t)\right)\right\}}{\left[\sum_{m} \delta\left(r_{i}(t)-r_{m}(t)\right)\right]^{2}} \\
&- \frac{-\sum_{m} \nabla_{r_{m}}\left\{\delta\left(r_{i}(t)-r_{m}(t)\right) * R V\left(r_{i}, t\right)\right\}}{\left[\sum_{m} \delta\left(r_{i}(t)-r_{m}(t)\right)\right]^{2}} \\
&= \frac{\sum_{n} \delta\left(r_{i}(t)-r_{n}(t)\right) \sum_{m} \nabla_{r_{i}}\left\{\delta\left(r_{i}(t)-r_{m}(t)\right) *\left[d_{t} r_{m}(t)-d_{t} r_{i}(t)\right]\right\}}{\left[\sum_{m} \delta\left(r_{i}(t)-r_{m}(t)\right)\right]^{2}}
\end{aligned}
$$

$$
\begin{aligned}
& -\frac{\sum_{m} \nabla_{r_{m}}\left\{\delta\left(r_{i}(t)-r_{m}(t)\right) * R V\left(r_{i}, t\right)\right\}}{\left[\sum_{m} \delta\left(r_{i}(t)-r_{m}(t)\right)\right]^{2}} \\
& =\frac{\nabla_{r_{i}} R V\left(r_{i}, t\right)}{\left[\sum_{m} \delta\left(r_{i}(t)-r_{m}(t)\right)\right]}-\frac{\sum_{m} \nabla_{r_{m}}\left\{\delta\left(r_{i}(t)-r_{m}(t)\right) * R V\left(r_{i}, t\right)\right\}}{\left[\sum_{m} \delta\left(r_{i}(t)-r_{m}(t)\right)\right]^{2}},
\end{aligned}
$$

where we have used relations

$\nabla_{r_{i}} \delta\left(r_{i}(t)-r_{m}(t)\right)=-\nabla_{r_{m}} \delta\left(r_{i}(t)-r_{m}(t)\right) ;$

$\nabla_{r_{i}} d_{t} r_{i}(t)=d_{t} \nabla_{r_{i}} r_{i}(t)=d_{t} 1=0$

$\nabla_{r_{m}} \sum_{n}\left\{\delta\left(r_{i}(t)-r_{n}(t)\right) *\left[d_{t} r_{m}(t)-d_{t} r_{n}(t)\right]\right.$

$=\sum_{n} \nabla_{r_{m}}\left\{\delta\left(r_{i}(t)-r_{n}(t)\right) *\left[d_{t} r_{m}(t)-d_{t} r_{n}(t)\right]\right\}=0 ;$

$\sum_{n}\left\{\delta\left(r_{i}(t)-r_{n}(t)\right) *\left[d_{t} r_{m}(t)-d_{t} r_{n}(t)\right]\right\}$

$=\left[d_{t} r_{m}(t)-d_{t} r_{i}(t)\right] \sum_{n} \delta\left(r_{i}(t)-r_{n}(t)\right)+R V\left(r_{i}(t), t\right) ;$

$\nabla_{r_{m}}\left\{\delta\left(r_{i}(t)-r_{m}(t)\right) *\left[d_{t} r_{m}(t)-d_{t} r_{i}(t)\right] * \sum_{n} \delta\left(r_{i}(t)-r_{n}(t)\right)\right\}$

$=\sum_{n} \delta\left(r_{i}(t)-r_{n}(t)\right) * \nabla_{r_{m}}\left\{\delta\left(r_{i}(t)-r_{m}(t)\right) *\left[d_{t} r_{m}(t)-d_{t} r_{i}(t)\right]\right\} ;$

The term "zero-temperature" means that at any position, all particles at same time-space point have a same velocity and hence

$R V\left(r_{i}(t), t\right) \equiv \sum_{m} \delta\left(r_{i}(t)-r_{m}(t)\right) *\left[d_{t} r_{m}(t)-d_{t} r_{i}(t)\right]=0$,

$u\left(r_{i}(t), t\right)=d_{t} r_{i}(t)$, 
Thus, above long formulus will suggest

$\nabla_{r_{i}} u\left(r_{i}(t), t\right)=\frac{\nabla_{r_{i}} 0}{\left[\sum_{m} \delta\left(r_{i}(t)-r_{m}(t)\right)\right]^{2}}$

$-\frac{\sum_{m} \nabla_{r_{m}}\left\{\delta\left(r_{i}(t)-r_{m}(t)\right) * 0\right\}}{\left[\sum_{m} \delta\left(r_{i}(t)-r_{m}(t)\right)\right]^{2}}=0$.

This clearly explains why "zero-temperature" means the absence of the so-called convective term.

\section{CONFLICT OF INTEREST}

The author confirms that this article content has no conflicts of interest.

\section{ACKNOWLEDGEMENTS}

Declared none.

\section{REFERENCES}

Esarey E, Sprangle P, Krall J, Ting A. Overview of Plasma-based accelerator concepts. IEEE Trans Plasma Sci 1996; 24: 252-88.

[2] Esarey E, Schroeder CB, Leemans WP. Physics of laser-driven plasma-based accelerators. Rev Mod Phys 2009; 81: 1229-86.

[3] (a) Akhiezer AI, Polovin RV. Theory of wave motion of an electron plasma. Zh Eksp Teor Fiz 1956; 30: 915-28. (b) Akhiezer AI, Polovin RV. Theory of wave motion of an electron plasma. Sov Phys JETP 1956; 3: 696-705.

[4] Noble RJ, Cole F, Donaldson R. In Proceedings of the $12^{\text {th }}$ International Conference on High Energy Accelerators, Eds, Batavia, Illinois, 1984.

[5] (a) Rosenzweig JB. Nonlinear plasma dynamics in the plasma wake-field accelerator. Phys Rev Lett 1987; 58: 555-58, (b) Rosenzweig JB. Multiple-fluid models for plasma wake-field phenomena. Phys Rev A 1989; 40: 5249-55.

[6] Katsouleas TC. Physical mechanisms in the plasma wake-field accelerator. Phys Rev A 1986; 33: 2056-64.

[7] Bernstein IB, Greene JM, Kruskal MD. Exact nonlinear plasma oscillations. Phys Rev 1957; 108: 546-50.

[8] Sprangle P, Esarey E, Ting A. Nonlinear interaction of intense laser pulse in plasmas. Phys Rev A 1990; 41: 4463-9.

[9] Verma PS, Sengupta S, Kaw PK. Residual Bernstein-GreeneKruskai-like waves after one-dimensional electron wave breaking in a cold plasma. Phys Rev E 2012: 86: 016410.

[10] Wolf U, Schamel H. Wake-field generation by the ponderomotive memory effect. Phys Rev E 1997; 56: 4656-64.

[11] Ghizzo A, Johnston TW, R/veill| Bertrand TP, Albrecht-Marc M. Stimulated-Raman-scatter behavior in a relativistically hot plasma slab and an electromagnetic low-order pseudocavity. Phys Rev E 2006; 74: 046407.
[12] (a) Breaking of Large Amplitude Plasma Oscillations. Phys Fluids 1971; 14: 1402-6. (b) Coffey TP. Large amplitude relativistic plasma waves. Phys Plasmas 2010; 17: 052303.

[13] Schroeder CB, Esarey E, Shadwick BA, Leemans WP. Trapping, dark current, and wave breaking in nonlinear plasma waves. Phys Plasmas 2006; 13: 033103. (b) Schroeder CB, Esarey E. Relativistic warm plasma theory of nonlinear laser-driven electron plasma waves. Phys Rev E 2010; 81: 056403.

[14] Schroeder CB, Esarey E, Shadwick BA. Warm wave breaking of nonlinear plasma waves with arbitrary phase velocities. Phys Rev E 2005; 72: 055401 .

[15] Yazdanpanah J, Anvari A, Samimi J. Warm relativistic fluid description of the laser wake field accelerator. Phys Plasmas 2009; 16: 023104.

[16] Trines RMGM, Norreys PA. Wave-breaking limits for relativistic electrostatic waves in a one-dimensional warm plasma. Phys Plasmas 2006; 13: 123102.

[17] Barnard JJ, Lund SM. Intense Beam Physic. Benjamin Inc 2004.

[18] Humphries S. Charged Particle Beams. John-Wiley 1990.

[19] (a) Davidson RC. Physics of Nonneutral Plasmas. Imperial College Press 2001, (b) Davidson RC. Theory of Nonneutral Plasmas. Addison-Wesley Pub 1989.

[20] Chen FF. Introduction to plasma physics. Plenum press 1974.

[21] Ichimaru S. Basic principles of plasma physics. Benjamin Inc 1973.

[22] Klemperer O, Barnett ME. Electron Optics. Cambridge University Press 1971.

[23] Wolnik H. Optics of Charged Particles. North-Holland 1981.

[24] Chao AW. Physics of Collective Instabilities in High Energy Accelerators. John-Wiley 1993.

[25] Lawson JD. Physics of Charged Particle Beams. Oxford University Press 1988 .

[26] (a) Chao AW, Pitthan R, Tajima T, Yeremian D. Space charge dynamics of bright electron beams. Phys. Rev. ST-AB 2003; 6: 024201. (b) Davidson RC. Three-dimensional kinetic stability theorem for high-intensity charged particle beams. Phys Plasmas 1998; 5: 3459-3468. (c) Batygin YK, Self-consistent analysis of three-dimensional uniformly charged ellipsoid with zero emittance. Phys Plasmas 2001; 8: 3103-6.

[27] Lund SM, Takashi K, Davidson RC. Generation of initial kinetic distributions for simulation of long-pulse charged particle beams with high space-charge intensity. Phys Rev ST-AB 2009; 12: 114801 .

[28] Krall NA, Trivelpiece AW. Principles of plasma physics. New York: McGraw-Hill 1977.

[29] Lin H, Shen BF Xu ZZ. Phase space coherent structure of charged particles system. Phys Plasmas 2011; 18: 062107.

[30] Lin H, Shen BF, Xu ZZ. Real Spatial Shapes and Phase Space Structure of 3-D Nonlinear Electrostatic Plasma Wave. The Open Plasma Phys J 2012; 5: 23-35.

[31] Lin H, Liu CP, Shen BF and Xu ZZ, Macroscopic and Microscopic Structure of Electromagnetic Wakefield. The Open Plasma Phys 2014: 7: 1-12.

[32] Lin H, Exact solutions of macroscopic self-consistent electromagnetic fields and microscopic distribution of VlasovMaxwell system. arxiv.org e-print 1402.7072. 